\title{
Educación alimentaria como puente entre extensión, docencia e investigación: el caso de una escuela para adultos de Santa Fe
}

\author{
María Antonella Paravano \\ Graduada de la Facultad de Bioquímica y \\ Ciencias Biológicas. Universidad Nacional \\ del Litoral, Argentina. \\ antonellaparavano@gmail.com
}

\author{
Liliana del Valle Ortigoza \\ liliortigoza@gmail.com \\ Gimena Valeria Dezar \\ gdezar@unl.edu.ar
}

Universidad Nacional del Litoral,

Argentina.
Investigación y extensión universitaria / Intervenciones
RECEPCIÓN: 22/06/18

ACEPTACIÓN FINAL: 25/06/18

\section{Resumen}

Plantear una mayor articulación e integración de las tres funciones básicas de la universidad constituye no solo un ideal sino una necesidad a la que se debe prestar atención. Este artículo propone visibilizar la articulación de proyectos de extensión y trabajos de investigación de alumnos con el fin de promover una sinergia y convergencia entre ambos. Desde ese lugar, se relata el trabajo de una tesinista de la Licenciatura en Nutrición de la Universidad Nacional del Litoral, que desarrolló su investigación en el marco de una Práctica de Extensión de Educación Experiencial en la que participaron docentes, alumnos y tutores de la Licenciatura, junto a alumnos, docentes y directivos de una escuela de educación media para adultos de Santa Fe. Las acciones coordinadas de los distintos actores posibilitaron estrechar vínculos con la comunidad y acercar a los estudiantes universitarios a situaciones reales. La investigación realizada permitió valorar facilitadores y obstaculizadores presentes en este proceso.

Palabras clave: educación alimentaria nutricional, adultos, Prácticas de Extensión de Educación Experiencial, tesina.
Food education as a bridge between extension, teaching and research: the case of a school for adults in Santa Fe

\section{Abstract}

Proposing a greater articulation and integration of the three basic functions of the university constitutes not only an ideal, but a need to which attention must be paid. This article proposes to visualize the articulation of extension projects and student research works, in order to promote a synergy and convergence between both. From that place, the work of a Nutrition Degree's advanced student of the Universidad Nacional del Litoral is related, that developed a investigation in the frame of an Extension Practice of Experiential Education in which participated teachers, students and tutors of the Degree, together with students, teachers and directors of a middle school for adults in Santa Fe. The coordinated actions of the different actors made it possible to strengthen links with the community, bringing university students closer to real situations. The research made it possible to assess facilitators and obstacles present in this process

Keywords: nutritional food education, adults, Extension Practices of Experiential Education, thesis.
A educação alimentar como ponte entre extensão, ensino e pesquisa: o caso de uma escola para adultos em Santa Fé

\section{Resumo}

Propor uma maior articulação e integração das três funções básicas da universidade constitui não apenas um ideal, mas uma necessidade para a qual se deve prestar atenção. Este artigo descreve uma pesquisaconduzida por estudantes universitarios de Nutrição (LN) da Universidad Nacional do Litoral (UNL), que foi desenvolvidano âmbito da Prática Educação Experimental de Extensão (PEEE). O projeto envolveu professores, estudantes e tutores LN, junto com a comunidade educativa de uma Escola de Esino Medio para Jovens e Adultos (EEMP) Santa Fe. O artigo torna visível a articulação entre extensão e pesquisa, promovendo sinergia e convergência entre ambas. As ações conjuntas dos diferentes atores possibilitaram o fortalecimento dos laços com a comunidade, aproximando os estudantes universitários às situações reais. A pesquisa possibilitou avaliar facilitadores e obstáculos presentes neste processo.

Palavras-chave: educação alimentar nutricional, adultos, Práticas de Extensão de Educação Experiencial, tesina.

Para citación de este artículo: Paravano, M.A, Ortigoza, L. del V. y Dezar, G.V. (2018). Educación alimentaria como puente entre extensión, docencia e investigación: el caso de una escuela para adultos de Santa Fe. +E: Revista de Extensión Universitaria, 8(9), julio-diciembre, 240-250. doi: 10.14409/ extension.v8i9.Jul-Dic.7860. 


\section{Introducción}

Las funciones sustantivas de la universidad como institución social son la docencia, la investigación y la extensión, cada una de ellas con un propósito específico. La integración de estas funciones universitarias pretende dar respuesta a las demandas de formación científica, tecnológica, humanística y al compromiso de generar conocimientos aplicables que contribuyan a satisfacer las necesidades de globalización y sustentabilidad social (González Agudelo, 2006).

En este trabajo se propone evidenciar la articulación entre docencia, extensión e investigación, desarrollada por un equipo extensionista de la Facultad de Bioquímica y Ciencias Biológicas (FBCB) de la Universidad Nacional del Litoral (UNL), trabajando de manera colaborativa con instituciones secundarias de la ciudad de Santa Fe, incluidas dentro de la Educación Permanente de Jóvenes y Adultos en la Argentina (EPJA).

Plantear una mayor articulación e integración de las tres funciones básicas de la universidad constituye no solo un ideal, que en la actualidad conserva su plena vigencia, sino una necesidad a la que se debe prestar mayor atención. Es un desafío que invita a la búsqueda creativa y urgente, tanto de posibilidades de intercambio entre cada una de esas funciones universitarias como de espacios de convergencia, donde esas funciones logren armonizarse (Lozano Cazabianca et al., 2012).

En esta dirección, el Plan de Desarrollo Institucional (PDI) de la UNL aprobado por Asamblea Universitaria correspondiente al período 2010-2019 propone que:

"la extensión es una función esencial de nuestra Universidad que promueve el diálogo permanente con el Estado -en sus diferentes jurisdicciones- con otras instituciones educativas y científico tecnológicas del sistema provincial y nacional, con las organizaciones de la sociedad civil y de la producción en la búsqueda de soluciones a los problemas del contexto, lo que confiere a estas acciones una alta pertinencia social [...] Además se destaca que [...] la integración entre la Extensión y la Enseñanza nos posiciona desde una perspectiva en la que los saberes culturales y los conocimientos académicos-científicos se ponen en juego con otros saberes y conocimientos que posee la sociedad, lo que posibilita analizar y abordar los problemas a partir de una concepción y comprensión de la complejidad". (UNL, 2010:s/n)

Con respecto a la temática, en los Lineamientos Generales para la Gestión FBCB/Escuela Superior de Sanidad período 2014-2018 —que tienen un correlato con el PDI de la UNLse hace referencia a la profundización de la inserción social de la Facultad, potenciando la interacción con los diferentes actores sociales y gubernamentales, buscando proyectos comunes y fomentando la integración.

En el marco del Proyecto y Acción del PDI "Fortalecimiento de la Educación Experiencial en el currículo universitario" ${ }^{1}$ de la Secretaría de Extensión de la UNL, se concibe a las Prácticas de Extensión como "actividades curriculares realizadas por los alumnos que impliquen diversos niveles de interacción e intervención en el medio social, cultural y productivo", y que se enmarcan bajo la modalidad de Educación Experiencial.

1) Las PEEE fueron aprobadas en el año 2007 por una resolución del Consejo Superior (Res. CS N² 274/07). 
A través de las Prácticas de Extensión de Educación Experiencial (PEEE), se propone al alumno desarrollar experiencias que le permitan poner en diálogo y enriquecer las habilidades y conocimientos teóricos que poseen; evaluar el estado de situación de procesos sociales y plantear soluciones posibles ante determinados problemas que se le presentan.

Considerado lo expresado por Boffelli y Sordo (2016), la intervención social que proponen las PEEE ancla en una construcción de conocimiento, lo que implica un conocimiento que se produce desde el hacer mismo, es decir, el estudiante aprende en situación. Esta intervención se funda en una concepción alejada de la enseñanza de meros contenidos teóricos y de aprendizaje lineal y aplicativo y brega por la idea de un aprendizaje que se construye desde el hacer mismo y en diálogo con diferentes campos del saber. Posicionar la intervención desde esta premisa implica corrernos de lo tradicional y abrirnos al desafío de otros modos de enseñar y aprender (Boffelli y Sordo, 2016).

Coincidiendo con las autoras, nos abrimos al desafío de otros modos de enseñar y aprender, y al proyecto de PEEE: "Promoción de Educación Alimentaria en Jóvenes y Adultos: desafío conjunto Universidad-Comunidad" le sumamos -integramos, potenciamos y podríamos agregar muchos otros términos- la realización de tesinas de grado de los alumnos de la Licenciatura en Nutrición (LN) de la FBCB. Lo planteamos como un doble desafío: por un lado, la apertura a otros modos de enseñar y aprender en situaciones reales y contextualizadas y, por otro, una mayor articulación e integración de las tres funciones básicas de la universidad. Esto nos posibilita el intercambio y la convergencia entre extensión, docencia e investigación, y a seguir trabajando en esta dirección para lograr armonizar las funciones a partir de ponerlas en diálogo.

Durante esta PEEE, llevada adelante por las cátedras de "Educación para la Salud y Economía Familiar", "Práctica Profesional" y "Seminario de Trabajo Final", se desarrollaron dos tesinas de la carrera de LN. Una de las tesinistas llevó a cabo el trabajo de campo de la investigación en la Escuela secundaria nocturna $N^{\circ} 440$, y la otra investigadora en la Escuela de Enseñanza Media Para Adultos (EEMPA) № 1305, ambas instituciones pertenecientes a la EPJA, de la ciudad de Santa Fe.

En el presente artículo intentamos hacer visible la articulación de la $\operatorname{PEEE}^{2}$ y la tesina desarrollada en el EEMPA $N^{\circ} 1305$ como un modo de promover una sinergia y convergencia entre ambos.

Luego de recuperar la conceptualización de la extensión y sus prácticas en el marco de la UNL, abordaremos la Educación Alimentaria Nutricional (EAN) enfocándonos en su importancia y relación con la EPJA. Desde este lugar, relataremos los vínculos universidad-comunidad, teniendo como puente el trabajo de investigación realizado.

\section{La importancia de la EAN en jóvenes y adultos, abordado desde las PEEE}

Dentro de la educación para la salud, se encuentra el campo de la EAN, cuyo fin es promover la modificación de la conducta alimentaria de la sociedad hacia patrones más saludables. 
Para ello es imprescindible el desarrollo de programas de educación nutricional, cuyo objetivo es la promoción de la salud mediante el aprendizaje, adecuación y aceptación de hábitos alimentarios saludables, de acuerdo con la propia cultura alimentaria y los conocimientos científicos en materia de nutrición que se tienen hasta el momento (Gil, 2010).

Desde esta perspectiva, es relevante la definición de EAN que hace Contento:

"La combinación de estrategias educativas, acompañadas de ambientes favorables, diseñadas para facilitar la adopción voluntaria de conductas, comportamientos y elecciones adecuadas en alimentación y nutrición para mantener o mejorar la salud y el bienestar". (2011:176)

Esta definición atiende al enfoque tridimensional de la nutrición que distingue al ser humano y a sus problemáticas de salud desde una perspectiva integral que involucra el contexto biológico, social y ambiental (Macias et al., 2009).

La EAN debe aplicarse en todas las fases de la vida, aunque tiene especial importancia en la edad escolar y adolescencia, pues son las etapas donde se forman hábitos. También resulta de interés en la edad adulta, induciendo modificaciones positivas de conductas relacionadas con la salud para conseguir finalmente una calidad de vida adecuada (Gil, 2010).

Por otro lado, la Food and Agriculture Organization (FAO, 2017) reconoce que es una prioridad realizar intervenciones de nutrición en el ámbito de las instituciones educativas, y considera a la escuela como un lugar ideal para la enseñanza de conocimientos básicos en alimentación, nutrición y salud. Esto puede crear beneficios que se extienden más allá de las aulas y el recreo, para mejorar la salud y el bienestar nutricional de hogares y comunidades.

En la FBCB de la UNL, específicamente en la carrera de Licenciatura en Nutrición, desde el equipo extensionista venimos haciendo hincapié en la EAN abordando las distintas etapas de la vida y trabajando colaborativamente con diferentes instituciones y organizaciones de la sociedad.

Durante el año 2016, el equipo extensionista trabajó desde el marco de un proyecto PEEE con eje en EAN promoviendo hábitos saludables en jóvenes adultos de dos escuelas de la ciudad de Santa Fe: la Escuela № 440 —secundaria nocturna-y la EEMPA № 1305 , teniendo en cuenta el entorno donde se desarrollan.

La inclusión de los nuevos modos y escenarios de los grupos desfavorecidos y vulnerables, como son la mayoría de los alumnos que acceden y concurren a las escuelas nocturnas, se convierten en una nueva realidad, en una nueva posibilidad y, fundamentalmente, en un nuevo desafío.

En Argentina, la EPJA se ha ocupado de que toda la población no escolarizada pueda cumplimentar sus estudios, tanto en niveles primarios como secundarios. Sin embargo, la población con vulnerabilidad social ha sido la que ha adquirido mayores derechos y la posibilidad de alfabetizarse en el sistema formal y no formal.

La EPJA a lo largo de la historia ha ido performando su campo conforme al contexto político y social y se ha caracterizado de manera particular. En la actualidad, nos encontramos con una modalidad diversa en sujetos que la componen, lo que permite identificar un universo heterogéneo con un sinnúmero de particularidades vinculadas a la propia trayectoria que traza la historia de vida de los alumnos (EPJASF, 2014). 
La provincia de Santa Fe avanza en nuevas modalidades de enseñanza innovadora y flexible y ofrece a aquellos interesados en completar sus estudios la posibilidad de optar por un servicio educativo que se va adaptando a sus necesidades (Resoluciones № 140/14; № 251/15. Ministerio de Educación de la Provincia de Santa Fe).

Actualmente, las EEMPA — que en otros tiempos abrían sus puertas a hombres y mujeres grandes que, por algún motivo, no habían podido terminar sus estudios- reciben a chicos solo unos años mayores que los que van a la secundaria común. El motivo: es más breve y admite sostener paralelamente un trabajo o una "changa".

El campo de la educación para jóvenes y adultos se orienta a atender a una población que se caracteriza, por un lado, por no haber podido acceder a la escuela y, por otro, (en caso de acceder a la escuela) por no haber finalizado los estudios y retomar la escolaridad luego de sucesivos fracasos. En consecuencia, la modalidad EEMPA se consolidó como una oferta educativa resultado de los fracasos de la escolarización inicial, de la condición educativa y cultural de los sectores sociales subalternos, y tuvo un carácter fuertemente compensatorio que no logró resolver el "círculo vicioso" de la desigualdad social (Brusilovsky y Cabrera, 2006).

La institución educativa donde se desarrolló el PEEE cuenta con una población de jóvenes adultos proveniente de contextos socioculturales diversos y con características escolares de fracaso.

La educación de los adultos tiene características particulares, ellos son conscientes de sus necesidades educativas, tienen la madurez como para seleccionar los medios y la forma de aprender, y se asume que tienen tiempo limitado y deben equilibrar las demandas de la familia, el trabajo y la educación (Ongay et al., 2013).

Una proporción muy importante de la vida corresponde a la etapa de adulto y durante ella el estilo de vida saludable es primordial para lograr un envejecimiento exitoso que lleve a una vejez con calidad; es a través de la EAN que podemos trabajar en mejorar estilos de vida poco saludables.

\section{La PEEE como nexo entre docencia, investigación y extensión}

Retomando lo anterior, la educación experiencial puede ser utilizada como una estrategia de enseñanza y como un componente del currículo. La primera implica un aprender haciendo y el segundo, demanda reflexión sobre el hacer. Esta reflexión "es la que transforma la simple experiencia en experiencia de aprendizaje" y es la que "hace educativa la experiencia", en términos de Alicia Camilloni (2013:15-17).

Desde ese marco, a partir del año 2013, docentes de distintas disciplinas de la carrera de Licenciatura en Nutrición propusimos integrar docencia, investigación y extensión, a través de PEEE, proyectos de Acciones de Extensión al Territorio (AET) y proyectos de Extensión de Interés Social (PEIS).

Participamos de manera colaborativa al interior del equipo docente, promoviendo espacios de diálogo, debate y reflexión sobre propósitos, desarrollo y maneras de llevar a cabo los diversos proyectos, surgidos a partir del interés manifiesto de distintos actores sociales.

En el caso de esta experiencia que exponemos, se trabajó desde un proyecto PEEE con eje en EAN. "Promoción de Educación Alimentaria en Jóvenes y Adultos: desafío conjunto 
Universidad-Comunidad", con dos instituciones educativas de enseñanza para adultos de la ciudad de Santa Fe. La tesina objeto de este artículo se llevó a cabo en una de las instituciones participantes.

En los jóvenes y adultos, el aprendizaje se produce en forma significativa, dado que la persona en situación de aprendizaje combina la información nueva con la que ya posee y reajusta y reconstruye ambas informaciones en este proceso. La estructura de los conocimientos previos condiciona los nuevos conocimientos y experiencias, y estos, a su vez, modifican y reestructuran aquellos.

En diálogo con las autoridades de uno de los establecimientos escolares su vicedirectora expresó:

"Pienso que estamos asistiendo a la construcción de una nueva escuela secundaria, en donde priorizamos los vínculos que permitan una educación para la paz y la no violencia, y desde ese lugar acceder a los conocimientos y saberes necesarios para esta época". (Entrevista, diario El Litoral, sábado 23 de agosto de 2014)

En esta dirección, teniendo en cuenta la situación de vulnerabilidad de los jóvenes y adultos participantes, se realizó un diagnóstico de situación preliminar a cargo de alumnos de la carrera de Licienciatura en Nutrición, quienes pudieron relacionarse con los estudiantes, el contexto escolar, hábitos e intereses en cuanto a nutrición y alimentación saludable, como primera etapa en el abordaje del proyecto realizado.

Nos fuimos aproximando así al conocimiento del contexto, lo que permitió un aprendizaje desde el hacer mismo.

Formaron parte de esta iniciativa las cátedras de $5^{\circ}$ año de Licienciatura en Nutrición -Educación para la Salud y Economía Familiar, Práctica Profesional y Seminario de Trabajo Final - y 80 alumnos avanzados de la carrera que cursaban dichas asignaturas y/o desarrollaban su Trabajo Final de Licenciatura en Nutrición (TFLN, comúnmente conocido como "tesina").

La experiencia llevada a cabo hizo énfasis en el papel de la educación como transformadora de la sociedad, reconociendo su participación en la formación integral de los jóvenes adultos del EEMPA y de los futuros profesionales de Licienciatura en Nutrición.

Los alumnos de las asignaturas comentadas y los que desarrollaron el trabajo de campo de su tesina pudieron adaptarse al territorio con facilidad porque se instrumentó una actividad de presentación, "Mateada", donde pudieron generar primeros vínculos con los estudiantes de las escuelas participantes e indagar sobre sus intereses con relación al proyecto.

En el desarrollo de los talleres, con la participación activa de los estudiantes mediante actividades lúdico-didácticas, se logró trabajar con entusiasmo y asistieron alumnos del último ciclo de las escuelas secundarias nocturnas.

En el desarrollo de la PEEE se pudo evidenciar:

- La colaboración del total de los participantes por llevar adelante la propuesta.

- La escucha y el diálogo del equipo extensionista con los participantes de las instituciones educativas.

- El interés por la temática de alimentación y salud por parte de los estudiantes y su entusiasmo. 
La experiencia fortaleció el trabajo en equipo y, dentro del proyecto, acercó a los participantes, a través de la EAN, a promover hábitos de vida saludable. Desde ese lugar se fortalecieron vínculos universidad-comunidad, mediante los cuales los estudiantes universitarios pudieron aprender en contexto y desarrollar competencias para su profesión futura.

En coincidencia con Subirats (2002), creemos que la experiencia realizada se concibió como un espacio que posibilitó construir de forma compartida la idea de calidad de vida. Es así que todos los actores se vieron implicados, asumieron compromisos y riesgos y pudieron establecer conexiones entre grupos y reconocer intereses comunes y complementarios.

Asimismo, de acuerdo con Menéndez (2013), el equipo extensionista sostiene que es posible profundizar la integración de la extensión con la docencia y la investigación en tanto se comprende el valor estratégico que poseen la educación y el conocimiento para la formación de ciudadanos libres y comprometidos y para la construcción de sociedades más inclusivas y democráticas.

Se trata de generar procesos de formación que posibiliten una experiencia de apropiación autónoma de este saber, experiencia que se asienta en vinculación con otras vivenciadas durante la formación (Bernik et al., 2007).

\section{Aportes de la tesina a los participantes directos del EEMPA}

La PEEE surgió debido al interés manifiesto de la EEMPA № 1305 y el interés del equipo extensionista de la carrera de Licienciatura en Nutrición para desarrollar estrategias didácticas con eje en EAN en la población joven-adulta. Tuvo como propósitos fortalecer acciones en la comunidad educativa orientadas a contribuir en la promoción de una alimentación que contemple cantidad, calidad, armonía y adecuación, así como también a la formación y compromiso social de los estudiantes universitarios.

En el marco de este proyecto, se desarrolló la tesina denominada "Educación Alimentaria Nutricional y Tecnologías de la Información y la Comunicación en una escuela para adultos de Santa Fe". ${ }^{3}$ La investigación tuvo lugar a partir del segundo cuatrimestre del año 2016.

Inicialmente, mediante la recuperación de los insumos trabajados durante la primera etapa de la PEEE, la investigadora llevó a cabo el primer encuentro correspondiente a su tesina en la institución educativa, donde se explicó el trabajo de investigación a realizar y se aplicó una encuesta diagnóstica. El objetivo de la misma fue conocer y describir los hábitos alimentarios de los alumnos del EEMPA y relacionarlos con algunos mensajes de las Guías Alimentarias para la Población Argentina (GAPA).

En segunda instancia, luego del análisis y reflexión sobre los resultados de la encuesta diagnóstica, se diseñaron, planificaron e implementaron talleres lúdico-didácticos con utilización de TIC como herramienta facilitadora para promover hábitos alimentarios saludables. Cabe mencionar que, a los fines de este artículo, no se desarrollarán los talleres realizados.

Con posterioridad, se aplicó nuevamente la encuesta inicial para poder identificar y comparar los conocimientos y prácticas adquiridas sobre hábitos alimentarios. En última instancia de la investigación, se realizó una encuesta de opinión con los estudiantes que participaron de las diferentes etapas. 
En el EEMPA № 1305 se trabajó con 100 jóvenes adultos desde 18 hasta de 55 años, principalmente varones, que provenían en su mayoría de los distritos norte, este y oeste de la ciudad de Santa Fe (Argentina). Los estudiantes —durante la realización de los talleres de EAN - se encontraban cursando el segundo ciclo del EEMPA en diferentes asignaturas.

Después de las intervenciones educativas, se destacaron las siguientes modificaciones en los hábitos alimentarios de los participantes, en comparación con las respuestas que los mismos estudiantes dieron en la encuesta diagnóstica:

- Aumentó el número de comidas recomendado por día (4 o más) de 32 a $65 \%$.

- Se incrementó el número de alumnos que comenzaron a desayunar (de 40 a 72 \%).

- Aumentó el consumo de pan blanco, integral y tostadas, de los alumnos durante el desayuno de 39 a $51 \%$, y se observó una disminución en el resto de los alimentos ricos en grasas saturadas y azúcares simples, como galletitas dulces y saladas (de 35 a $19 \%$ ), facturas y bizcochos de un 20 a $17 \%$.

- Con respecto a la bebida diaria con la que desayunan, luego de las intervenciones hubo un aumento en el consumo de leche de 28 a $38 \%$, en el yogurt de un 15 a un $28 \%$; y en cuanto a las infusiones, hubo una disminución considerable, de un 82 a un $34 \%$.

- Con referencia a los grupos de alimentos que están presentes en la alimentación diaria, incrementaron el consumo del grupo "Carnes y huevos" (de 58 a $93 \%$ ), además hubo un aumento considerable en los grupos "Verduras y frutas" (de 58 a $86 \%$ ) y "Lácteos" (de 55 a $81 \%)$; en menor medida aumentó el consumo del grupo "Legumbres, cereales, papa, pan y pastas" (de 67 a $80 \%$ ).

- En cuanto a las bebidas que consumen a diario los participantes, la de mayor predominio fue el agua antes y después de las intervenciones, incrementando levemente su consumo luego de los talleres (de un 38a $48 \%$ ).

En el ámbito educativo, la Promoción de Salud tiene como tarea fundamental mejorar el nivel de conciencia y capacidad de acción, tanto individual como colectiva, a través de sus proyectos.

Lo anterior se evidenció en la encuesta de opinión realizada al finalizar los talleres sobre EAN, en las que los participantes pudieron valorar dichos talleres, hacer comentarios y proponer modificaciones para optimizar futuras prácticas, retroalimentando así la experiencia.

Se identificaron y analizaron "frases y/o párrafos significativos" mediante las cuales los estudiantes expresaron sus consideraciones acerca de los talleres efectuados:

- "Siempre es importante mantener una vida saludable."

- "Refresco de memoria para mantener una vida sana y sostener los hábitos saludables."

- "Hay que ser consciente a la hora de alimentarnos."

- "Seguir viviendo mejor, comer sano."

- "Estuvo interesante, me gustó poder expresarme y educarme cada vez mejor" .

- "Los talleres me dejaron enseñanza y reflexión."

- "La buena alimentación y la actividad física son saludables para nuestra vida".

- "Me informaron sobre los alimentos y sus beneficios. Cómo, cuándo y qué comer."

- "Que la gente se eduque y aprenda a conocer su cuerpo, cómo funciona y cómo debe alimentarse y saber lo que come." 
- "Me enseñaron la importancia que la buena nutrición tiene en nuestra vida."

- "Me gustaron, para poder aprender y saber cómo cuidarme."

- "Alimentarse bien para un cuerpo saludable."

- "Buenos hábitos, vida más fácil."

- "Cuidar nuestra alimentación para tener una buena salud. Muy innovadoras y divertidas."

- "Alimentarse de forma adecuada y moverse con frecuencia favorece nuestra salud."

De las frases significativas mencionadas por los alumnos se puede inferir que para ellos fue positivo e interesante aprender sobre alimentación saludable. Se ha demostrado que la educación nutricional en el entorno escolar es capaz de influir en los conocimientos sobre alimentación y nutrición.

Las intervenciones favorecieron los procesos de conocimiento respecto de la incorporación de hábitos saludables y los resultados indican la fuerza que adquiere el trabajo en conjunto cuando es visibilizado mediante acciones contundentes y se refuerza la importancia de la extensión como vía para fortalecer los vínculos universidad-sociedad desde la promoción de salud, con énfasis en el desarrollo de entornos saludables.

La EAN con la utilización de actividades lúdico-didácticas es una de las estrategias para propiciar una educación más dinámica y participativa, donde los alumnos se involucren individual y grupalmente. Cuando se potencia la EAN en el ámbito escolar, se pueden mejorar directamente la salud y la nutrición de los jóvenes adultos y promover hábitos alimenticios saludables en ellos y en sus familias.

Un importante facilitador del trabajo de campo fue la dinámica implementada, ya que al interactuar con un grupo de personas de edades diferentes surgieron muchas preguntas que dieron lugar a una participación activa, compromiso con los talleres, un intercambio enriquecedor tanto para los estudiantes del EEMPA como para la investigadora.

Como obstáculo, debemos mencionar el tiempo con el que contamos para desarrollar los talleres y luego llevarlos a cabo; también surgieron desajustes entre la planificación estipulada y los tiempos reales de la institución educativa, extendiéndose la finalización de la tesina.

En esta dirección, se debe continuar trabajando en facilitar la adopción voluntaria de conductas, comportamientos y elecciones adecuadas en alimentación y nutrición para mantener o mejorar la salud y el bienestar, en la medida en que debe ser una persona activa frente a su realidad, capaz de contribuir al desarrollo propio, al de su familia y de su comunidad.

\section{Reflexiones finales}

En coincidencia con Rafaghelli (2013), sostenemos que toda acción de extensión implica una intervención en un espacio determinado, en un territorio específico y en un entramado de relaciones particulares.

A través de las PEEE, se propone al alumno realizar actividades en las que, a partir de esa conexión con la práctica, puedan desarrollar experiencias que plantean poner en diálogo, en situación auténtica, las habilidades y los conocimientos teóricos que poseen, evaluar el estado de situación de los procesos sociales, enriquecer esos conocimientos y habilidades, realizar aportes a posibles soluciones e identificar nuevos problemas. 


\section{¿Qué es posible promover desde este lugar?}

El estudiante universitario, mediante la práctica en terreno, se enfrenta a la necesidad social desde el plano académico, profesional, investigativo, establece los mecanismos de solución, lo que provoca la transformación del estudiante y de la comunidad universitaria como contexto de formación fundamental en el cual se sintetiza la relación universidad-comunidad.

Esta formación integral debe apuntar a la transformación que ocurre en el sujeto en los ámbitos de formación, en donde, en sus diferentes interacciones, reconstruye y se desarrolla culturalmente y corrobora la influencia de lo social en lo individual.

\section{¿Por qué la educación alimentaria como puente?}

Si se asume la extensión universitaria como un proceso integrador y dinámico, presente en cada uno de los eslabones estructurales de la universidad, que permite expresar el vínculo social más amplio, proponemos como puente la EAN ya que se manifiesta como una necesidad sentida por la sociedad en su conjunto.

Consideramos que la educación alimentaria se convierte en un desafío para todos los actores para abordarla en toda su amplitud y complejidad. Adhiriendo a Macias et al. (2009), se trata de un enfoque tridimensional de la nutrición, de manera integral, que involucra los contextos biológico, social y ambiental.

En ese marco, se trata de promover en la comunidad educativa estilos de vida saludable y en los estudiantes universitarios afianzar competencias para el futuro profesional, íntimamente vinculadas con el contexto real.

\section{Referencias bibliográficas}

Bernik, J.; Baraldi, V.; Díaz, N.; Stringhini, M.E. (2007). De cómo recuperar la intelectualidad, lo colectivo y lo utópico. indicios para pensar la formación de los docentes. Santa Fe, Argentina: Ediciones UNL.

Boffelli, M. y Sordo, S. (2016). Fundamentos y perspectivas desde donde pensar la integración de la docencia y la extensión. Revista +E, 6 (6), 16-23. Santa Fe, Argentina: Ediciones UNL.

Brusilovsky, S.; Cabrera, M. (2006). Educación escolar de adultos. Buenos Aires: Noveduc.

Camilloni A. (2013). La inclusión de la educación experiencial en el curriculum universitario. En Menéndez G. et al. Integración docencia y extensión. Otra forma de aprender y de enseñar. Santa Fe: Ediciones UNL.

Contento, I.R. (2011). Nutrition Education: Linking Theory, Research, and Practice, 17(1), 176-179.

EPJASF (2014). Situación de la Educación Permanente de Jóvenes y Adultos de la Provincia de Santa Fe. Ministerio de Educación de la provincia de Santa Fe. Dirección General de Información y Evaluación Educativa. Santa Fe.

Food and Agriculture Organization (FAO) (2017). Página web: http://www.fao.org/school-food/en/

Gil Hérnandez, A.D.; Maldonado Lozano, J.; Martínez de Victoria Muñoz, E. (2010). Tratado de Nutrición: Nutrición humana en el Estado de Salud. Vol. 3, p. 550. Madrid: Médica Panamericana. 2da. ed.

González Agudelo, E.M. (2006). La investigación formativa como una posibilidad para articular las funciones universitarias de la investigación, la extensión y la docencia. Rev. Educ. Pedag., (18), 101-109.

Lozano Casabianca, G.A.; Ochoa Hoyos, A.M.; Restrepo Mesa, S.L. (2012). La articulación entre investigación, docencia y extensión en un programa universitario de Nutrición y Dietética. Perspect. Nutr. Humana,(14), 71-83. Universidad de Antioquia. Medellín, Colombia. 
Macias, A.I.; Quintero, M.L.; Camacho, E.J. y col. (2009). La tridimensionalidad del concepto de nutrición: su relación con la educación para la salud. Rev. Chil. Nutr., 36(4), 1129-1135. Recuperado de http://www.scielo. cl/scielo.php?script=sci_arttext\&pid=S0717-75182009000400010

Menéndez, G. et al. (2013). Integración docencia y extensión. Otra forma de aprender y de enseñar. Santa Fe: Ediciones UNL.

Ministerio de Educación de la Provincia de Santa Fe (2014). Resolución № 140/14.

(2015). Resolución №251/15.

Rafaghelli, M. (2013). La dimensión pedagógica de la extensión. Menéndez, G. et al. Integración docencia y extensión. Otra forma de aprender y de enseñar. Santa Fe: Ediciones UNL.

Universidad Nacional del Litoral (2007). Resolución Honorable Consejo Superior № 274. Santa Fe, Argentina. (2010). Resolución Asamblea Universitaria No 2: Plan de Desarrollo Institucional de la Universidad Nacional del Litoral 2010-2019. Universidad Nacional del Litoral. Santa Fe, Argentina.

Ongay, E.; Saravia, L.; Cortada, D. et al. (2013). Capacitación de funcionarios de servicios de alimentación y nutrición (LATIn). Recuperado de http://escritura.proyectolatin.org/educacion-alimentaria-y-nutricional-en-el-marco-de-la-educacion-para-la-salud/capacitacion-de-funcionarios-de-servicios-de-alimentacion-y-nutricion/ (consultado el 11/06/18).

Subirats, J. (Coord.). (2002). Educación y territorio. El factor proximidad y de comunidades en las políticas educativas. En Gobierno Local y Educación. La importancia del territorio y la comunidad en el papel de la escuela (pp. 23-41). Barcelona: Ariel. 